\title{
La formation des futurs médecins généralistes aux gestes $d$ 'urgence et de premier secours reste sous-optimale en France
}

Monsieur,

Les médecins généralistes sont souvent amenés à intervenir en tant que premier maillon de la chaîne des secours, que ce soit sur leur lieu d'exercice dans le cadre des soins primaires, dans un véhicule des sapeurs pompiers ou dans la vie quotidienne [1]. De ce fait, à l'issue de leur cursus de formation spécialisée, ils devraient maîtriser les gestes de premiers secours. Nous rapportons les résultats d'une enquête effectuée auprès d'étudiants inscrits en diplôme d'études spécialisées (DES) de médecine générale, visant notamment à vérifier dans quelle mesure un tel objectif de formation professionnelle était atteint.

En juillet 2011, un questionnaire auto-administré a été envoyé à 334 étudiants de la Faculté de médecine de l'Université d'Angers (France), respectivement inscrits en DES de médecine générale $(n=212)$ ou en année de thèse pour le diplôme d'état de docteur en médecine $(n=122)$. Le questionnaire, qui avait été élaboré avec l'aide des responsables universitaire du département de médecine générale, en s'appuyant sur des référentiels nationaux de compétence [2], recueillait la perception des étudiants relativement à leur aptitude à réaliser les gestes de premiers secours et les gestes médicaux d'urgence, explorait leurs connaissances déclaratives dans le domaine du secourisme à l'aide de questions construites à partir des recommandations de pratique et des résultats de conférences de consensus [2, 3], et sollicitait enfin leur niveau de satisfaction relativement à la formation aux gestes de premiers secours et d'urgence qu'ils avaient reçue et leurs souhaits à cet égard. Par ailleurs, il permettait de colliger un certain nombre d'informations personnelles (formations reçues, avancement dans le cursus en fonction du nombre de stages validés ou non, faculté de médecine d'origine, projet professionnel).

Parmi les 151 étudiants (45\%) ayant répondu, $15(10 \%)$ indiquaient ne pas avoir reçu de formation aux premiers secours et gestes d'urgences. La perception de leur aptitude à réaliser les gestes de premiers secours et gestes médicaux d'urgence était très variable selon les situations auxquelles ils avaient pu être ou non confrontés. Seuls $3 \%$ et $5 \%$ avaient respectivement été confrontés à la réanimation cardiopulmonaire (RCP) ou à la prise en charge d'une douleur thoracique aiguie au cabinet de médecine générale mais $15 \%$ avaient eu l'occasion de réaliser une aspiration bronchique, d'utiliser un défibrillateur ou de poser une canule de Guedel. Parmi les étudiants qui possédaient une trousse d'urgence, $60 \%$ d'entre eux ne disposaient pas de diazépam ou de clonazépam.

Leurs connaissances déclaratives relatives aux gestes de premiers secours et aux gestes médicaux d'urgence étaient très variables puisque le taux de bonnes réponses pour les indications de la position latérale de sécurité était de $94 \%$ mais qu'il n'était que de $10 \%$ pour la prise en charge d'une hémorragie d'un membre lorsque le pansement compressif s'avérait insuffisant. Aucune caractéristique individuelle n'était associée à la maîtrise des gestes de secourisme, à l'exception de l'ancienneté, associée à une meilleure mâ̂trise du cycle massages/insufflations $(p<0,002)$ et de la manœuvre d'Heimlich $(p<0,03)$. 
Le taux d'insatisfaction vis-à-vis de la formation pratique reçue était élevé $(67,7 \%)$ chez les internes en cours de DES, voire très élevé chez les étudiants en cours de thèse ( $80 \%)$. Il était moindre à l'égard de la formation théorique $(32,4 \%)$. Pour $83 \%$ des répondants, la remise à niveau devait être obligatoire; selon $41 \%$ des étudiants, elle pouvait avoir lieu tous les deux ans. Vingt-deux étudiants évoquaient la nécessité que la formation au secourisme soit répétée dans le cadre chaque cycle de formation, pour ne pas perdre les automatismes et trois évoquaient la nécessité d'une évaluation pratique, d'un contrôle des connaissances et d'une formation continue pour les médecins généralistes, la formation pouvant se faire à l'hôpital au sein de l'équipe soignante mais aussi de manière adaptée, centrée sur l'apprentissage de la capacité à gérer seul la situation d'urgence au cabinet. Les difficultés évoquées pour le maintien des acquis étaient : le changement régulier des recommandations, le manque de temps pour se former soi-même et le manque de pratique liée au filtre du dispositif territorial de régulation de la permanence des soins.

En dépit de ses limites, liées notamment au caractère purement déclaratif des connaissances vérifiées et de l'absence d'évaluation des habiletés et capacités effectivement maîtrisées, cette enquête rapporte des résultats étonnant puisque $10 \%$ des répondants déclarent ne pas avoir de formation aux gestes de premiers secours comme aux gestes d'urgence et que seuls $63 \%$ indiquent avoir reçu une formation équivalente à l'attestation de formation aux gestes et soins d'urgence (AFGSU), alors que celle-ci est devenue obligatoire au cours du deuxième cycle des études médicales en France depuis 2007 [4].

Les gestes d'urgences les moins maîtrisés sont les gestes les plus techniques et les moins fréquents (moins de la moitié des étudiants y a été confrontée) : réalisation d'un accouchement, intubation et repositionnement d'une canule de trachéotomie (45 à $57 \%$ des étudiants ne se sentent pas aptes à les pratiquer) [5]. La réanimation cardio-pulmonaire, la prise en charge d'une douleur thoracique et l'utilisation de morphine en titration sont des gestes connus par plus de neuf étudiants sur 10. La prise en charge d'un choc anaphylactique, l'utilisation du défibrillateur automatisé externe et l'utilisation du diazépam ou du clonazépam par voie intra rectale sont des actes qui semblent connus (par au moins deux tiers des étudiants interrogés) mais les situations correspondantes sont peu rencontrées (par moins d'un tiers des répondants seulement). Pour ce qui est de la pose d'une perfusion intra veineuse, il n'y a pas de différence significative entre les différentes promotions d'étudiants.

Le stage aux urgences a une influence significative sur l' aptitude à prendre en charge un œdème aigu pulmonaire, une douleur thoracique aigue, à préparer un aérosol en cas de crise d'asthme aigu, à utiliser le diazépam ou le clonazépam intra-rectal en cas de crise d'épilepsie, à poser une canule de Guedel et à réaliser une aspiration bronchique par voie endo nasale ou endo trachéale ; il s'agit-là de gestes spécifiques aux urgences. Le stage chez le praticien a peu d'influence sur l'acquisition des gestes d'urgence; l'enquête montre cependant une différence significative pour la prise en charge d'une douleur thoracique aigüe. Le fait d'avoir effectué un stage aux urgences est associé à une meilleure perception d'aptitude à effectuer des gestes d'urgences. Le projet d'installation, respectivement en campagne ou en ville, n'est associé à aucune différence significative des résultats au questionnaire. Le fait d'avoir validé les premier et deuxième cycles à la faculté de médecine d'Angers ou dans une autre faculté française n'a pas d'incidence statistiquement significative sur les résultats.

Les gestes de premiers secours et les gestes médicaux d'urgence dont la maîtrise était explorée dans l'enquête avaient été identifiés à partir des dernières recommandations et conférences de consensus disponibles lors de l'envoi du questionnaire. Les plus forts pourcentages de mauvaises réponses se rapportent à la technique de désobstruction des voies aériennes en cas d'inhalation de corps étranger totalement obstructif ou syndrome de pénétration, la réanimation cardio-pulmonaire chez l'enfant et les plaies hémorragiques (62 à $90 \%$ de mauvaises réponses), qui correspondent à des activités peu fréquentes dans la pratique. Pour les autres questions, le taux de mauvaises réponses varie de 28 à $6 \%$ [2,3], un tel résultat illustrant les difficultés rencontrées par les secouristes face au changement des recommandations et la nécessité de proposer des recyclages réguliers. Par 
exemple, depuis 2010 l'International Liaison Committee on Resuscitation recommande un rythme de massage cardiaque externe supérieur ou égal à 100 et inférieur à 120/min alors qu'il était préalablement de 100/min, ce changement étant effectif dans les recommandations de secourisme depuis le premier juillet 2012 [6].

Concernant la satisfaction des étudiants vis-à-vis de la formation reçue en matière de premiers secours et de gestes d'urgence, il est singulier de constater que les étudiants en cours d'année de thèse s'estiment les moins bien formés, notamment au plan pratique, alors qu'ils ont en principe davantage d'expérience.

L'expérience d'un stage au service des urgences est tenue pour être primordiale pour se préparer à affronter des situations de stress et d'indécision dans l'exercice futur, au cabinet de médecine générale mais certaines données suggèrent que la perception d'efficacité personnelle peut être altérée par la confrontation à des situations d'échec [7], ce qui souligne la nécessité que les étudiants y bénéficient d'une supervision pédagogique de qualité.

La majorité des répondants souhaite une remise à niveau tous les deux ans, ce qui équivaut à un rappel au cours du premier, du deuxième et du troisième cycle puis de manière régulière pendant l'activité professionnelle (sous forme de stages ou d'activités de formation médicale continue). Les récents et fréquents changements de recommandations en matière de secourisme mettent en difficultés les étudiants qui ne semblent pas avoir le temps de mettre à jour leurs connaissances de manière autonome, comme l'illustre le moindre niveau d'aptitude perçue en matière de secourisme par les étudiants en année de thèse.

En conclusion, pris dans leur ensemble et malgré leurs limites, les résultats de notre enquête suggèrent que, en dépit de dispositions réglementaires explicitement prescriptives en matière de formation aux gestes d'urgence et de premier secours tout au long du cursus, la formation des futurs médecins généralistes reste à cet égard probablement sous-optimale en France.

\footnotetext{
Clarisse AUDOUIN ${ }^{1}$ Guillaume BOUZILLE ${ }^{2}$ Monique LELOUP ${ }^{3}$ Serge FANELLO ${ }^{2}$
}

${ }^{1}$ Service d'accueil et d'urgence, Centre hospitalier universitaire d'Angers, France

${ }^{2}$ Département universitaire de santé publique, Unité de formation et de recherche de médecine d'Angers, 49045 Angers Cedex 1, France Mailto : sefanello@chu-angers.fr

${ }^{3}$ Service Départemental d'incendie et de secours de la Mayenne, Laval, France

\section{Références}

1. Code de Déontologie Médicale. Article 9. Assistance à personne en danger (article R.4127-9 du code de la santé publique). 2012 [On-line] Disponible sur: http://www.conseil-national.medecin.fr/article/ le-code-de-deontologie-medicale-915

2. Ministère de l'Intérieur. Référentiel Technique PSC1 «Prévention et secours civiques niveau 1 ». 2009 ( $2^{\text {ème }}$ édition) [On-line] Disponible sur : www.interieur.gouv.fr

3. Ministère de l'Intérieur. Référentiel national de compétences de sécurité civile. "Premiers secours en équipe de niveau $1 » 2010$ ( $3^{\text {ème }}$ édition). [On-line] Disponible sur : www.interieur.gouv.fr

4. Arrêté du 20 avril 2007 relatif à la formation aux gestes et soins d'urgence au cours des études médicales, odontologiques et pharmaceutiques (NOR: SANP0752363A). Journal Officiel de la République Française $2007\left(n^{\circ}\right.$ 107) : texte $n^{\circ}$ 60. [On-line] Disponible sur : http://www.legifrance.gouv.fr/

5. Dick ML, Schluter P, Johnston C, Coulthard M. GP's perceived competence and comfort in managing medical emergencies in southeast Queensland. Aust Fam Physician 2002;31:870-5.

6. Hazinski MF, Nolan JP, Billi JE, Böttiger BW, Bossaert L, de Caen AR, et al. Part 1: Executive Summary: 2010 International Consensus on Cardiopulmonary Resuscitation and Emergency Cardiovascular Care Science With Treatment Recommendations. Circulation 2010;122(16 Suppl 2):S250-75.

7. Pelaccia T, Delplancq H, Triby E, Bartier J-C, Leman $\mathrm{C}$, Dupeyro J-P. Impact of training periods in the emergency department on the motivation of health care students to learn. Med Educ 2009;43:462-69. 